\title{
Magnetic Screening in Accreting Neutron Stars
}

\author{
Andrew Cumming, Ellen Zweibel ${ }^{1}$, and Lars Bildsten ${ }^{2}$ \\ Institute for Theoretical Physics, Kohn Hall, University of California, Santa Barbara, CA 93106; \\ cumming@itp.ucsb.edu; zweibel@solarz.colorado.edu; bildsten@itp.ucsb.edu
}

Submitted to The Astrophysical Journal, 8th February 2001

\begin{abstract}
We investigate whether the magnetic field of an accreting neutron star may be diamagnetically screened by the accreted matter. We assume the freshly accumulated material is unmagnetized, and calculate the rate at which the intrinsic stellar magnetic flux is transported into it via Ohmic diffusion from below. For very high accretion rates $\dot{M}$ (larger than the Eddington rate $\dot{M}_{\text {Edd }}$ ), Brown and Bildsten have shown that the liquid ocean and outer crust of the neutron star are built up on a timescale much shorter than the Ohmic penetration time. We confirm this result and go further to calculate the screening, both in this limit and at lower accretion rates, where the screening declines. Considering the liquid ocean and outer crust, we find that the Ohmic diffusion and accretion timescales are equal for $\dot{M} \approx 0.1 \dot{M}_{\text {Edd }}$.

We calculate the one-dimensional steady-state magnetic field profiles, and show that the magnetic field strength decreases as one moves up through the outer crust and ocean by $n$ orders of magnitude, where $n \approx \dot{M} / 0.02 \dot{M}_{\mathrm{Edd}}$. We show that these profiles are unstable to buoyancy instabilities when $B \gtrsim 10^{10}-10^{11} \mathrm{G}$ in the ocean. This provides a new limit to the possible strength of any buried field. Most steadily accreting neutron stars in low-mass X-ray binaries in our Galaxy accrete at rates where screening would be effective if the accretion and magnetic geometry is as we portray. However, we cannot definitively state that the magnetic fields of these objects are screened at high levels. If screened, then the underlying field will emerge after accretion halts, on a timescale of only 100-1000 years, set by the Ohmic diffusion time across the outer crust. The definitive statement we can make is that magnetic screening is ineffective for $\dot{M}<10^{-2} \dot{M}_{\text {Edd }}$, so that, no matter how the accreted material joins onto the star, the underlying stellar field should always be evident. In this respect, we point out the only known persistently-pulsing accreting X-ray millisecond pulsar, SAX J1808.4-3658, has an accretion rate of $\dot{M} \sim 10^{-3} \dot{M}_{\text {Edd }}$, far below the realm where magnetic screening can play a role.
\end{abstract}

\footnotetext{
${ }^{1}$ Permanent address: JILA, U. Colorado, Boulder CO 80309

${ }^{2}$ Also: Department of Physics, University of California, Santa Barbara, CA 93106
} 
Subject headings: accretion - magnetic fields — pulsars: individual: SAX J1808.4-3658 — stars: neutron - x-rays: binaries

\section{Introduction}

Accreting neutron stars fall generally into two classes (see White, Nagase, \& Parmar 1995). Those in high mass X-ray binaries (HMXBs) are X-ray pulsars, with dipole magnetic fields $B \sim 10^{12} \mathrm{G}$, large enough to disrupt the accretion flow and channel it onto the polar caps. Most of the neutron stars in low mass X-ray binaries (LMXBs), however, show no direct evidence for a magnetic field $\left(B \lesssim 10^{10} \mathrm{G}\right.$ ). The accretion disk is believed to extend almost to the stellar surface, allowing these neutron stars to be spun up to millisecond periods by accretion. Thus LMXB neutron stars are favored candidates for the progenitors of millisecond radio pulsars (Bhattacharya 1995), and as such are expected to have magnetic fields $B \sim 10^{8}-10^{9} \mathrm{G}$.

Only one LMXB so far has shown a pulse indicative of magnetically-controlled accretion, the newly-discovered transient SAX J1808.4-3658. This source shows persistent $401 \mathrm{~Hz}$ pulsations, making it the first accretion-powered millisecond X-ray pulsar (Wijnands \& van der Klis 1998; Chakrabarty \& Morgan 1998). Interpreting the pulsations as disruption of the accretion flow by a magnetosphere leads to an estimated magnetic field strength of $10^{8}-10^{9} \mathrm{G}$ (Psaltis \& Chakrabarty 1999), suggesting that this source will become a millisecond radio pulsar once accretion ceases (Bildsten \& Chakrabarty 2001).

The origin of such low external magnetic fields $\lesssim 10^{10} \mathrm{G}$ is not understood, but is believed to be directly connected with accretion of matter onto the neutron star. This suggestion is based on the observations that isolated neutron stars show no evidence for dramatic field decay, and that low field radio pulsars occur almost exclusively in binaries (Bhattacharya \& Srinivasan 1995). Utilizing binary evolution calculations, Taam \& van den Heuvel (1986) studied a number of systems, finding that magnetic field strength decreases with the amount of accreted matter, although Wijers (1997) argues against this interpretation. A number of different mechanisms for achieving this reduction in magnetic field strength have been proposed (see Bhattacharya \& Srinivasan 1995 for a review).

One possibility that could explain the low dipole fields and the lack of pulsations from these sources is that the external dipole magnetic field is "buried" or "screened" by the accreted matter. Bisnovatyi-Kogan \& Komberg (1974) suggested that pulsars in binaries may have low magnetic fields because of this effect. Romani $(1990,1995)$ outlined a picture in which matter initially accretes onto the polar cap, eventually spreads under the weight of the accumulated matter, and advects magnetic field inwards. He proposed that the magnetic flux would be trapped in the crust and screened by further accretion of diamagnetic matter.

In this paper, we test this possibility. We consider accretion of unmagnetized matter onto a neutron star with a buried magnetic field, and ask whether the field remains buried, or whether 
the newly accreted material becomes magnetized faster than the rate at which it is accreted, allowing the intrinsic stellar field to "leak out". We study the competition between downwards compression and advection by accretion at the local rate, $\dot{m}$, and upwards Ohmic diffusion. We carry out this calculation for the ocean and outer crust of the neutron star that are in steady-state once accretion has been occurring for an extended period. We show that, in steady-state, the magnetic field strength decreases through the outer crust and ocean by $n \approx \dot{m} / 0.02 \dot{m}_{\text {Edd }}$ orders of magnitude, where $\dot{m}_{\text {Edd }}$ is the Eddington accretion rate. We show that these profiles are unstable to buoyancy instabilities in the ocean when $B \gtrsim 10^{10}-10^{11} \mathrm{G}$, giving a new limit to the strength of any buried field.

We find that screening could be effective at the accretion rates of most steadily accreting neutron stars. Unfortunately, because of the simplified magnetic field and accretion geometry we adopt in this paper, we cannot definitively state that these objects have a screened field. However, if so, we show that after accretion ceases, we expect the dipole field to emerge on a timescale of 100-1000 years, set by the Ohmic diffusion time across the outer crust. It therefore seems unlikely that screening alone can explain the low magnetic fields of the millisecond radio pulsars.

Our robust conclusion is that it is not possible to diamagnetically screen the field in the crust for accretion rates $\lesssim 0.02 \dot{m}_{\text {Edd }}$. The lack of screening at low accretion rates may explain why SAX J1808.4-3658 is the only LMXB so far to show persistent pulsations. Consideration of both the observations and evolutionary state of this binary (Chakrabarty \& Morgan 1998; Bildsten \& Chakrabarty 2001) makes it clear that the time-averaged accretion rate is unusually low, $\dot{m} \approx 10^{-3} \dot{m}_{\text {Edd }}$, far below the realm where screening can occur.

Previous authors have considered the competition between accretion and Ohmic diffusion, but in different contexts. Brown \& Bildsten (1998) compared the Ohmic diffusion and advection times in the atmosphere, ocean and crust of the polar cap of an accreting X-ray pulsar. In this case, the accreted matter arrives on the star already threaded by the magnetic field, and may spread laterally if Ohmic diffusion is rapid enough. Considering magnetic field evolution in accreting neutron stars, Konar \& Bhattacharya (1997) assumed that the currents supporting the magnetic field were confined to the crust, and calculated the competing effects of Ohmic dissipation, and advection of magnetic flux into denser regions with longer dissipation times (and eventually into the superconducting core).

Another process which acts to transport magnetic flux outwards is thermomagnetic drift (e.g., Urpin \& Yakovlev 1980; Geppert \& Urpin 1994). In this paper, we consider the liquid ocean and solid crust of the neutron star, for which we find that thermomagnetic drift is unimportant; the magnetic field profile is set by the competition between Ohmic diffusion and accretion. However, in the $\mathrm{H} / \mathrm{He}$ layer which overlies the ocean, we find that thermomagnetic drift dominates the transport of magnetic field. The evolution of the magnetic field in this layer, which periodically undergoes a thermonuclear instability giving rise to Type I X-ray bursts, will be discussed in a separate paper. 
We start by describing the thermal structure of the ocean and crust, and what sets the electrical conductivity there $(\S 2)$. In $\S 3$, we discuss the evolution of the magnetic field under the joint action of accretion and Ohmic diffusion, and in $\S 4$ evaluate the characteristic timescales for these processes. In $\S 5$, we compute steady state magnetic profiles. We show that the magnetic field decreases with height in the ocean, by an amount that depends strongly on the local accretion rate, $\dot{m}$. In $\S 6$, we consider the stability of the steady-state profiles to magnetic buoyancy instabilities. We summarize our results and conclude in $\S 7$ with the implications for steadily and transiently accreting neutron stars.

\section{Microphysics and Structure of the Ocean and Crust}

In this section, we outline the structure of the outer layers of the neutron star, and describe how we calculate the electrical conductivity.

\subsection{Thermal Structure}

Most neutron stars in LMXBs accrete hydrogen and helium from their companions at rates $\dot{M} \sim 10^{-11}-10^{-8} M_{\odot} \mathrm{yr}^{-1}$. Since we are concerned with the outermost layers of thickness $\lesssim 100 \mathrm{~m}$, small compared to the radius, we adopt plane parallel coordinates and measure the accretion rate as a local rate $\dot{m}$ (units $\mathrm{g} \mathrm{cm}^{-2} \mathrm{~s}^{-1}$ ). The local Eddington accretion rate is $\dot{m}_{\mathrm{Edd}}=2 m_{p} c /(1+X) R \sigma_{\mathrm{T}}$, where $\sigma_{\mathrm{T}}$ is the Thomson scattering cross-section, $m_{p}$ is the proton mass, $c$ is the speed of light, $R$ is the stellar radius, and $X$ is the hydrogen mass fraction. We use the Eddington accretion rate for solar composition $(X=0.71)$ and $R=10 \mathrm{~km}$,

$\dot{m}_{\text {Edd }} \equiv 8.8 \times 10^{4} \mathrm{~g} \mathrm{~cm}^{-2} \mathrm{~s}^{-1}$, as our basic unit for the local accretion rate. For a $10 \mathrm{~km}$ neutron star, this corresponds to a global rate $\dot{M}_{\text {Edd }}=1.7 \times 10^{-8} M_{\odot} \mathrm{yr}^{-1}$.

The neutron star atmosphere is in hydrostatic balance as the accreted hydrogen and helium accumulates, because the downward flow speed $(v=\dot{m} / \rho)$ is much less than the sound speed $c_{s}$. The pressure varies with height as $d P / d z=-\rho g$, where the gravitational acceleration $g$ is constant in the thin envelope. We assume in this paper that the magnetic field does not play a role in hydrostatic balance, i.e. $d\left(B^{2} / 8 \pi\right) / d z \ll d P / d z$ (we check this assumption in $\S 6$ when we consider buoyancy instabilities). A useful variable is the column depth $y$ (units $\mathrm{g} \mathrm{cm}^{-2}$ ), defined by $d y \equiv-\rho d z$, giving $P=g y$. As the matter accumulates, a given fluid element moves to greater and greater column depth. We take $g_{14} \equiv g / 10^{14} \mathrm{~cm} \mathrm{~s}^{-2}=1.9$, the Newtonian gravitational acceleration for a $M=1.4 M_{\odot}$ and $R=10 \mathrm{~km}$ neutron star.

The accreted $\mathrm{H}$ and He burn at $y \approx 10^{8}-10^{9} \mathrm{~g} \mathrm{~cm}^{-2}$ and temperature $T \approx 2-5 \times 10^{8} \mathrm{~K}$, producing heavy elements which form the underlying ocean and crust. The microphysics of these layers has been discussed by Bildsten \& Cutler (1995) and Brown \& Bildsten (1998). The pressure in the ocean and outer crust is mostly provided by degenerate electrons. For the ions, we 
incorporate Coulomb corrections to the equation of state using the fit of Farouki \& Hamaguchi (1993). The crystallization point is determined by $\Gamma=(Z e)^{2} / k_{B} T a$, where $a$ is the interion spacing given by $4 \pi a^{3} n_{i} / 3=1, Z e$ is the ionic charge, $n_{i}$ is the ion number density (we assume only one species of ion is present). For typical conditions at top of the ocean, this is

$$
\Gamma=12 \rho_{5}^{1 / 3}\left(\frac{2}{T_{8}}\right)\left(\frac{Z}{30}\right)^{5 / 3}\left(\frac{2 Z}{A}\right)^{1 / 3},
$$

where $\rho_{5} \equiv \rho / 10^{5} \mathrm{~g} \mathrm{~cm}^{-3}, T_{8} \equiv T / 10^{8} \mathrm{~K}$, and the mass of each nucleus is $A m_{p}$. The ions solidify when $\Gamma=\Gamma_{m} \approx 173$ (Farouki \& Hamaguchi 1993), at a depth

$$
\begin{aligned}
y_{c r} \approx 2 \times 10^{13} \mathrm{~g} \mathrm{~cm}^{-2} & \left(\frac{T_{8}}{5}\right)^{4}\left(\frac{Z}{30}\right)^{-20 / 3} \\
& \left(\frac{1.9}{g_{14}}\right)\left(\frac{\Gamma_{m}}{173}\right)^{4},
\end{aligned}
$$

where we assume fully-relativistic electrons and use the $T=0$ Fermi energy.

We find the thermal structure of the ocean and crust by integrating the heat equation,

$$
\frac{d T}{d y}=\frac{3 \kappa F}{4 a c T^{3}}
$$

where $F$ is the outward heat flux, and $\kappa$ is the opacity. The heat flux is set by heat released from compression of matter by accretion, and from pycnonuclear reactions and electron captures deep in the crust (Brown \& Bildsten 1998). For our models, we assume a constant heat flux $F=150 \mathrm{keV}$ per nucleon. The opacity $\kappa$ is calculated as described by Schatz et al. (1999) and Brown (2000) for the crust (see discussion in $\S 2.2$ ). The temperature and pressure at the top of the ocean are set by $\mathrm{H} / \mathrm{He}$ burning in the upper atmosphere. For accretion rates $\dot{m}<\dot{m}_{\text {Edd }}$, the H/He burns unstably, and we take the temperature and column depth at the top of the ocean to be the conditions at He ignition, as calculated by Cumming \& Bildsten (2000). For $\dot{m}=\dot{m}_{\mathrm{Edd}}$, we use the steady-state burning model of Schatz et al. (1999), giving $y=10^{8} \mathrm{~g} \mathrm{~cm}^{-2}$ and $T=5 \times 10^{8} \mathrm{~K}$ at the top of the ocean. We take the composition of the ocean and crust to be nuclei with $Z=30$ and $A=60$. We arbitrarily stop our integrations at $y=10^{14} \mathrm{~g} \mathrm{~cm}^{-2}$, thus we include only the outer crust, above neutron drip.

The upper panel of Figure 1 shows the temperature profiles for $\dot{m}=0.01,0.1$ and $1 \dot{m}_{\mathrm{Edd}}$. For the 0.01 and $0.1 \dot{m}_{\text {Edd }}$ models, we mark the liquid-solid interface with a dot (the $\dot{m}_{\text {Edd }}$ model is still liquid at $y=10^{14} \mathrm{~g} \mathrm{~cm}^{-2}$ ). In the crust, we show two models for each accretion rate, each with a different choice for the thermal conductivity: phonon scattering or electron-ion scattering (see $\S 2.2$ for discussion of conductivity in the crust). 


\subsection{Electrical Conductivity}

In the relaxation time approximation, the electrical conductivity is $\sigma=n_{e} e^{2} / m_{\star} \nu_{c}$, or

$$
\sigma=7.6 \times 10^{20} \mathrm{~s}^{-1} \rho_{5}\left(\frac{2}{\mu_{e}}\right)\left(\frac{10^{16} \mathrm{~s}^{-1}}{\nu_{c}}\right)\left(\frac{m_{e}}{m_{\star}}\right)
$$

(e.g., Ziman 1964; Yakovlev \& Urpin 1980), where $n_{e}$ is the electron number density, $m_{\star}$ is the effective electron mass, $\mu_{e}$ is the mean molecular weight per electron, and $\nu_{c}$ is the electron collision frequency. The lower panel of Figure 1 shows the conductivity as a function of depth.

In the liquid ocean, electron-ion collisions set the electrical conductivity. We use the results of Yakovlev \& Urpin (1980), who give

$$
\sigma=\frac{8.5 \times 10^{21} \mathrm{~s}^{-1}}{Z \Lambda_{e i}} \frac{x^{3}}{1+x^{2}}
$$

where $\Lambda_{e i} \approx 1$ is the Coulomb logarithm, and $x \equiv p_{F} / m_{e} c$ measures the electron Fermi momentum $p_{F}$.

The conductivity in the solid crust depends on the level of impurity. Itoh \& Kohyama (1993) give the electron-impurity collision frequency as

$$
\nu_{e Q}=1.75 \times 10^{16} \mathrm{~s}^{-1} \frac{Q}{\langle Z\rangle} \Lambda_{e Q}\left(1+x^{2}\right)^{1 / 2},
$$

where $Q=\sum Y_{i}\left(Z_{i}-\langle Z\rangle\right)^{2} / \sum Y_{i}$ is the impurity parameter, $\langle Z\rangle=\sum Y_{i} Z_{i} / \sum Y_{i}=Y_{e} / \sum Y_{i}$ is the average charge (the number abundance of species $i$ is $Y_{i}=X_{i} / A_{i}$, where $X_{i}$ is the mass fraction), and the Coulomb logarithm is order unity. The other contribution to the conductivity is scattering with phonons, for which we adopt the results of Baiko \& Yakovlev (1995). For temperatures much greater than the Debye temperature $\left(T \gg \Theta_{D}=2.4 \times 10^{6} \mathrm{~K} x^{3 / 2}(2 Z / A)\right)$, they find

$$
\nu_{e p}=\frac{\alpha k_{B} T}{\hbar} 13 \Lambda_{e p}=1.24 \times 10^{18} \mathrm{~s}^{-1} T_{8} \Lambda_{e p},
$$

where $\alpha=1 / 137$ is the fine structure constant, and $\Lambda_{e p}$ is of order unity. The ratio of the impurity and phonon scattering frequencies is

$$
\frac{\nu_{e Q}}{\nu_{e p}} \approx 10^{-2} Q\left(\frac{3}{T_{8}}\right)\left(\frac{x}{60}\right)\left(\frac{30}{\langle Z\rangle}\right)\left(\frac{\Lambda_{e Q}}{\Lambda_{e p}}\right),
$$

where we choose typical conditions in the crust.

Previous studies have assumed a low level of impurity, $Q \ll 1$, as might be expected for a primordial crust. In this case phonon scattering dominates the conductivity. However, recent calculations of the products of $\mathrm{H} / \mathrm{He}$ burning in the atmosphere show that the impurity level is probably much greater. Schatz et al. (1999) calculated the ashes of steady-state H/He burning at 
local accretion rates $\dot{m} \gtrsim \dot{m}_{\mathrm{Edd}}$, and found $\langle Z\rangle=24$ and $Q \approx 100$. At such high impurity factors, it is not clear whether equation (5) is correct. Brown (2000) suggests that when $Q \approx\langle Z\rangle^{2}$, the appropriate conductivity is that for electron-ion scattering in the liquid.

In this paper, we show results for two limiting cases for the crust: a pure crust with only phonon scattering, and an impure crust with electron-ion scattering calculated as for the liquid. The conductivity in each case is shown in the lower panel of Figure 1. For each accretion rate, we plot the two solutions for the crust: for electron-ion scattering, the conductivity is continuous across the liquid-solid boundary; for phonon scattering, the conductivity changes discontinuously. 3 The difference in conductivity between these two cases is roughly a factor of 5 . In $\S 4$, we show that the impurity level has an important effect on magnetic field evolution in the outer crust.

\section{Evolution of the Magnetic Field}

We consider a simplified geometry for the magnetic field, a plane parallel model in which the magnetic field $\boldsymbol{B}$ depends only on depth. In this case, the vertical component of the magnetic field must be constant since $\boldsymbol{\nabla} \cdot \boldsymbol{B}=0$, and we set it equal to zero since we assume the accreted matter is unmagnetized. This leaves us with a purely horizontal field, $\boldsymbol{B}(z)=B(z) \hat{e}_{x}$ (we align our $x$-axis with the horizontal component of the field).

The magnetic field evolves according to Faraday's law

$$
\frac{\partial \boldsymbol{B}}{\partial t}=-c \boldsymbol{\nabla} \times \boldsymbol{E}
$$

where the electric field $\boldsymbol{E}$ is given by Ohm's law,

$$
\boldsymbol{E}=-\frac{\boldsymbol{v} \times \boldsymbol{B}}{c}+\frac{\boldsymbol{J}}{\sigma}
$$

The electric current is

$$
\boldsymbol{J}=\frac{c}{4 \pi} \boldsymbol{\nabla} \times \boldsymbol{B}=\hat{e}_{y} \frac{c}{4 \pi} \frac{\partial B}{\partial z},
$$

for a given profile $\boldsymbol{B}(z)$, and the downwards flow velocity is given by continuity as $\boldsymbol{v}=-v(z) \hat{e}_{z}=-(\dot{m} / \rho) \hat{e}_{z}$ (we define $v$ so that it is a positive quantity for the downwards flow). Thus $\boldsymbol{E}=E_{y} \hat{e}_{y}$, where

$$
E_{y}=\frac{v B}{c}+\frac{c}{4 \pi \sigma} \frac{\partial B}{\partial z}
$$

and equation (8) reduces to its $\hat{x}$ component

$$
\frac{\partial B}{\partial t}=\frac{\partial}{\partial z}\left[v B+\eta \frac{\partial B}{\partial z}\right]
$$

\footnotetext{
${ }^{3}$ Recent conductivity calculations of Potekhin et al. (1999) show that the conductivity is continuous across the melting point even in this case; however, the older results we adopt in this paper are sufficient for our purposes.
} 
where $\eta=c^{2} / 4 \pi \sigma$ is the magnetic diffusivity.

It is instructive to rewrite equation (12) as

$$
\rho \frac{D}{D t}\left(\frac{B}{\rho}\right)=\frac{\partial}{\partial z}\left(\eta \frac{\partial B}{\partial z}\right)
$$

where $D / D t=\partial / \partial t+\boldsymbol{v} \cdot \boldsymbol{\nabla}$ is the advective derivative and we have used the continuity equation $D \rho / D t=-\rho \boldsymbol{\nabla} \cdot \boldsymbol{v}$. Equation (13) clearly illustrates the different processes acting to change the magnitude of $B$. The left hand side describes advection and compression by the downwards flow; the right hand side describes Ohmic diffusion. Without diffusion, $B$ in a given fluid element would grow $\propto \rho$ as it was compressed.

In this paper, we calculate only steady state solutions of equation (12). Since we assume that the incoming material is unmagnetized, we set $E_{y}=0$, and equation (12) reduces to

$$
\eta \frac{d B}{d z}=-v B
$$

We rewrite this so that pressure is the independent variable; this makes the competition between advection and diffusion more transparent. Using hydrostatic balance, and introducing the pressure scale height $H \equiv y / \rho=-(d \ln P / d z)^{-1}$, equation (14) becomes

$$
\frac{d \ln B}{d \ln P}=\left(\frac{H^{2}}{\eta}\right)\left(\frac{\dot{m}}{y}\right)=\frac{t_{\mathrm{diff}}}{t_{\mathrm{accr}}},
$$

where we have defined

$$
t_{\text {diff }} \equiv \frac{H^{2}}{\eta}
$$

the characteristic Ohmic diffusion time across a scale height, and

$$
t_{\text {accr }} \equiv \frac{y}{\dot{m}},
$$

the characteristic accretion flow time across a pressure scale height. Equation (15) shows that, in steady-state, $t_{\text {diff }} / t_{\text {accr }}$ measures the ratio of pressure scale height to magnetic scale height. For example, if $t_{\text {diff }}>t_{\mathrm{accr}}$, a small magnetic scale height is required for Ohmic diffusion to balance accretion.

The magnetic profile is given by integrating equation (15), since all the quantities on the right hand side are known functions of pressure (or height). We find

$$
B(P)=B\left(P_{0}\right) S
$$

where $P_{0}$ is a reference pressure, and

$$
S \equiv \exp \left[-\int_{P}^{P_{0}} d \ln P\left(\frac{t_{\mathrm{diff}}}{t_{\mathrm{accr}}}\right)\right]
$$


is the amount by which the magnetic field changes due to screening from the overlying matter. We shall refer to the quantity $S$ as the magnetic screening factor.

The Ohmic diffusion time is independent of $B$ (see Goldreich \& Reisenegger 1992 for an interesting discussion). Thus equation (15) is linear in $B$, giving the profile of the magnetic field independent of its overall amplitude. In the field burial picture, the amplitude of $B$ in the crust is fixed by conserving the magnetic flux in the original dipole field. We do not calculate the details of the burial process in this paper; thus we treat the overall normalization of the magnetic field in the crust as a free parameter.

For our assumed field geometry, the Hall term in Ohm's law exactly vanishes. More generally, the magnitude of the Hall term depends on the ratio of the electron cyclotron frequency, $\omega_{e}=B e / m_{\star} c$, to the electron collision frequency, $\nu_{c}=\tau^{-1}$. For a typical value of $\nu_{c}$, this is

$$
\omega_{e} \tau=\left(\frac{B}{5.6 \times 10^{8} \mathrm{G}}\right)\left(\frac{10^{16} \mathrm{~s}^{-1}}{\nu_{c}}\right)\left(\frac{m_{e}}{m_{\star}}\right),
$$

where $m_{\star}=E_{e} / c^{2}$ is the effective mass of the electron. Typically, $\omega_{e} \tau>1$ requires $B \gtrsim 10^{11} \mathrm{G}$ in the ocean and crust; we show in $\$ 6$ that such large fields are buoyantly unstable. In addition, we neglect terms in Ohm's law due to thermomagnetic effects (e.g., Urpin \& Yakovlev 1980); these are unimportant in the ocean and outer crust.

\section{Accretion vs. Ohmic Diffusion}

In $\$ 3$, we showed that the steady-state magnetic profile depends directly on the ratio of accretion and Ohmic diffusion timescales. In this section, we evaluate this ratio, before presenting the steady-state profiles in 8 . Figure 2 shows the accretion and diffusion timescales as a function of depth, and Figure 3 shows $t_{\text {diff }} / t_{\text {accr }}$. We find that the diffusion time is longer than accretion time for $\dot{m} \gtrsim 0.1 \dot{m}_{\text {Edd }}$. We now use simple analytic estimates to understand these results, discussing the ocean (\$4.1) and crust (\$4.2) in turn.

\subsection{Ocean}

First, we evaluate $t_{\text {diff }}=4 \pi \sigma H^{2} / c^{2}$. The conductivity is given by equation (4). The degenerate electron pressure is $P_{e}=\left(m_{e} c^{2}\right)^{4} f(x) / 24 \pi^{2}(\hbar c)^{3}$ (Clayton 1983), or, in terms of column depth $y=P / g$,

$$
y_{8}=3.2 f(x)\left(\frac{1.9}{g_{14}}\right)
$$

where

$$
f(x)=x\left(2 x^{2}-3\right)\left(1+x^{2}\right)^{1 / 2}+3 \sinh ^{-1} x
$$


(Clayton 1983), and $y_{8} \equiv y / 10^{8} \mathrm{~g} \mathrm{~cm}^{-2}$. We write the density as $\rho=\mu_{e} n_{e} m_{p}$, giving $\rho_{5}=9.7 \mu_{e} x^{3}$. The scale height $H=y / \rho$ is thus

$$
H=170 \mathrm{~cm} \frac{f(x)}{x^{3}}\left(\frac{1.9}{g_{14}}\right)\left(\frac{2}{\mu_{e}}\right)
$$

giving

$$
t_{\mathrm{diff}}=10^{5} \mathrm{~s} \frac{f^{2}(x)}{x^{3}\left(1+x^{2}\right)} \Lambda_{e i}^{-1}\left(\frac{30}{Z}\right)\left(\frac{1.9}{g_{14}}\right)^{2}\left(\frac{2}{\mu_{e}}\right)^{2} .
$$

The accretion timescale is

$$
t_{\mathrm{accr}}=10^{4} \mathrm{~s} y_{8}\left(\frac{\dot{m}}{0.1 \dot{m}_{\mathrm{Edd}}}\right)^{-1}
$$

giving

$$
\begin{array}{r}
\frac{t_{\mathrm{diff}}}{t_{\mathrm{accr}}}=3 g(x) \Lambda_{e i}^{-1}\left(\frac{\dot{m}}{0.1 \dot{m}_{\mathrm{Edd}}}\right)\left(\frac{2}{\mu_{e}}\right)^{2} \\
\left(\frac{30}{Z}\right)\left(\frac{1.9}{g_{14}}\right),
\end{array}
$$

where $g(x) \equiv f(x) / x^{3}\left(1+x^{2}\right)$. Thus, $t_{\mathrm{diff}} \approx t_{\mathrm{accr}}$ for $\dot{m} \approx 0.1 \dot{m}_{\mathrm{Edd}}$, in agreement with Figure 3 .

In the non-relativistic and relativistic limits, the functions $f(x)$ and $g(x)$ take the limiting values

$$
f(x)= \begin{cases}8 x^{5} / 5 & x \ll 1 \\ 2 x^{4} & x \gg 1\end{cases}
$$

and

$$
g(x)=\left\{\begin{array}{ll}
8 x^{2} / 5 & x \ll 1 \\
2 / x & x \gg 1
\end{array} .\right.
$$

Thus

$$
\begin{array}{r}
\left(\frac{t_{\mathrm{diff}}}{t_{\mathrm{accr}}}\right)_{N R} \approx 3 y_{8}^{2 / 5} \Lambda_{e i}^{-1}\left(\frac{\dot{m}}{0.1 \dot{m}_{\mathrm{Edd}}}\right)\left(\frac{2}{\mu_{e}}\right)^{2} \\
\left(\frac{30}{Z}\right)\left(\frac{1.9}{g_{14}}\right)^{3 / 5},
\end{array}
$$

for $x \ll 1$, and

$$
\begin{array}{r}
\left(\frac{t_{\mathrm{diff}}}{t_{\mathrm{accr}}}\right)_{R} \approx y_{12}^{-1 / 4} \Lambda_{e i}^{-1}\left(\frac{\dot{m}}{0.1 \dot{m}_{\mathrm{Edd}}}\right)\left(\frac{2}{\mu_{e}}\right)^{2} \\
\left(\frac{30}{Z}\right)\left(\frac{1.9}{g_{14}}\right)^{5 / 4}
\end{array}
$$

for $x \gg 1$. These analytic estimates roughly agree with our numerical results, and explain the scaling of $t_{\text {diff }} / t_{\text {accr }}$ with depth; for small $x, t_{\text {diff }} / t_{\text {accr }}$ increases with depth, whereas for large $x$, $t_{\text {diff }} / t_{\text {accr }}$ decreases with depth. The total screening factor $S$ through the ocean is thus insensitive to the upper and lower boundaries. The maximum value of $t_{\text {diff }} / t_{\text {accr }}$ occurs for intermediate values of $y$, for which the electrons are partially relativistic $\left(x \sim 1, \rho \approx 10^{6}-10^{7} \mathrm{~g} \mathrm{~cm}^{-3}\right)$. 


\subsection{Crust}

We now turn to the solid crust. The behavior of $t_{\text {diff }} / t_{\text {accr }}$ is quite different in the crust, depending on whether we choose electron-ion scattering or phonon scattering. For electron-ion scattering, $t_{\text {diff }} / t_{\text {accr }} \propto g(x) \propto 1 / x$, decreasing with depth, as shown in Figure 3. For phonon scattering,

$$
t_{\mathrm{diff}}=2 \times 10^{5} \mathrm{~s} \frac{x^{4}}{T_{8}}\left(\frac{13 f_{e p}}{\Lambda_{e p}}\right)\left(\frac{1.9}{g_{14}}\right)^{2}\left(\frac{2}{\mu_{e}}\right)^{2},
$$

giving

$$
\begin{array}{r}
\left(\frac{t_{\mathrm{diff}}}{t_{\mathrm{accr}}}\right)_{p}=\frac{3}{T_{8}}\left(\frac{\dot{m}}{0.1 \dot{m}_{\mathrm{Edd}}}\right) \\
\left(\frac{1.9}{g_{14}}\right)\left(\frac{2}{\mu_{e}}\right)^{2}\left(\frac{13 f_{e p}}{\Lambda_{e p}}\right) .
\end{array}
$$

Thus $t_{\text {diff }} / t_{\text {accr }}$ is constant with depth in this case, in agreement with Figure 3. We see that, as suggested by Schatz et al. (1999), $t_{\text {diff }}$ is much smaller in an impure crust (electron-ion scattering) than a primordial crust (phonon scattering).

We do not calculate the conductivity of the inner crust, below neutron drip at $y \sim 10^{15_{-}}$ $10^{16} \mathrm{~g} \mathrm{~cm}^{-2}$. At these depths, Brown \& Bildsten (1998) found that $t_{\text {diff }}<t_{\text {accr }}$ for $\dot{m}<5 \dot{m}_{\text {Edd }}$ (see their Figure 9 for a continuation of our Figure 3 into the inner crust). Thus the inner crust does not give a significant contribution to the screening factor.

\section{Steady-State Profiles}

In section 4 , we compared the timescale for Ohmic diffusion with that for accretion. We now use the results of $\S 3$ to compute the steady-state magnetic profiles, by integrating equation (15).

Figure 1 shows the result for a range of accretion rates. The solid lines are for models with electron-ion scattering in the crust; for 0.01 and $0.1 \dot{m}_{\text {Edd }}$, the dotted lines show models with phonon scattering. Rather than fix the overall magnitude of the magnetic field, we plot the ratio of the magnetic field at each depth to the magnetic field at the base $\left(y=10^{14} \mathrm{~g} \mathrm{~cm}^{-2}\right)$. As expected from inspection of equation (15), we find that the magnetic and pressure scale heights are equal for $\dot{m} \approx 0.1 \dot{m}_{\mathrm{Edd}}$, the accretion rate at which $t_{\mathrm{diff}} \sim t_{\mathrm{accr}}(\S 4.1)$.

An analytic estimate of the screening factor for the ocean follows straightforwardly from the results of 8 . The ratio $t_{\text {diff }} / t_{\text {accr }}$ is given by equation (26). We then substitute $d \ln P=(d \ln f / d x) d x$ in equation (19), where $f(x)$ is given by equation (22) and

$d f / d x=8 x^{4} /\left(1+x^{2}\right)^{1 / 2}$. As we discussed in $\$ 4.1$, most of the contribution to $S$ comes from $x \sim 1$. Integrating, we find

$$
\ln S=2.4\left(\frac{\dot{m}}{0.01 \dot{m}_{\mathrm{Edd}}}\right)\left(\frac{30}{Z}\right)\left(\frac{1.9}{g_{14}}\right)
$$




$$
\left[\frac{1}{\left(1+x_{t}^{2}\right)^{1 / 2}}-\frac{1}{\left(1+x_{b}^{2}\right)^{1 / 2}}\right]
$$

where $x_{t}\left(x_{b}\right)$ is the value of $x$ at the top (bottom), and we take $\mu_{e}=2$ and $\Lambda_{e i}=1$. Note that equation (33) gives $\ln S$; the factor by which the magnetic field changes through the atmosphere is the exponential of this value.

Using the scalings of equation (33), and adopting the prefactor from our numerical results, we find that the magnetic field decreases by $n$ orders of magnitude through the ocean, where

$$
n \approx \frac{\dot{m}}{0.02 \dot{m}_{\mathrm{Edd}}}\left(\frac{Z}{30}\right)\left(\frac{g_{14}}{1.9}\right) .
$$

The major contribution to $n$ comes from the ocean. Thus the uncertainty in the conductivity of the crust (electron-ion or phonon scattering) changes $n$ by only a small amount, as shown by comparing the solid and dotted lines in Figure 4 .

\section{Buoyancy Instability}

We now investigate the stability of the steady-state magnetic profiles to buoyancy instabilities. We first consider interchange and Parker instabilities in $\$ 6.1$, before including the effects of thermal diffusion in $\$ 6.2$.

\subsection{Interchange and Parker Instabilities}

The simplest case to consider is the interchange instability in which a magnetic field line and associated fluid is lifted vertically, maintaining pressure balance with its surroundings. If the new density is less than that of the surrounding fluid, it is buoyantly unstable. Newcomb (1961) considered the stability of a horizontal magnetic field in a stratified atmosphere, pointing out that it is also important to consider Parker-type modes, in which the magnetic field lifts up, but the fluid flows back down the field lines. In the limit of long wavelength along the field (minimizing the energy used to bend the magnetic field lines), Newcomb showed that the Parker-type modes may be unstable even when the interchange modes are not, although with smaller growth rates.

Newcomb (1961) showed that the criterion for instability is

$$
\mathcal{A} \equiv \frac{d \ln \rho}{d z}+\frac{\rho g}{\Gamma_{1} P_{g}}>0,
$$

where $P_{g}$ is the gas pressure. Using the equation of hydrostatic balance, $d P / d z=$ $d\left(P_{g}+B^{2} / 8 \pi\right) / d z=-\rho g$, to substitute for $-\rho g$, we find instability if (see also Acheson 1979)

$$
\left(\frac{2}{\Gamma_{1}}\right)\left(\frac{B^{2}}{8 \pi P_{g}}\right)\left(-\frac{d \ln B}{d z}\right)>N^{2} / g
$$


where

$$
\frac{N^{2}}{g}=\frac{d \ln \rho}{d z}-\frac{1}{\Gamma_{1}} \frac{d \ln P_{g}}{d z}
$$

defines the Brunt Väisälä frequency $N^{2}$ (e.g., Hansen \& Kawaler 1994). Both thermal buoyancy and composition gradients may contribute to $N^{2}$ (e.g., Bildsten \& Cumming 1998). We assume a uniform composition in the ocean, so that $N^{2}$ has only a thermal piece,

$$
N^{2}=\frac{g}{H} \frac{\chi_{T}}{\chi_{\rho}}\left(\nabla_{\mathrm{ad}}-\frac{d \ln T}{d \ln y}\right)
$$

where $\chi_{Q} \equiv \partial \ln P / \partial \ln Q$ with the other independent thermodynamic variables held constant.

We see from equation (36) that the instability criterion depends on both how quickly the magnetic field decreases with height, and the ratio of magnetic to gas pressure, $B^{2} / 8 \pi P_{g}$. To understand this, first consider a magnetic field whose strength changes discontinuously with height. If the magnetic field above the discontinuity is smaller than that below, pressure continuity implies heavy fluid overlying light; a Rayleigh Taylor unstable density jump no matter how small $B^{2} / 8 \pi P_{g}$. On the other hand, if magnetic pressure dominates gas pressure, even a small magnetic field gradient may create an unstable density profile.

For the steady-state magnetic profile, the gradient $d \ln B / d z$ is given by $d \ln B / d \ln y=t_{\text {diff }} / t_{\text {accr }}$ (eq. 15]). Inserting this gradient into equation (36), we find the critical magnetic field strength $B_{c}$ required for instability of the steady-state profile is

$$
B_{c}^{2}=4 \pi \Gamma_{1} P_{g}\left(\frac{t_{\mathrm{accr}}}{t_{\mathrm{diff}}}\right)\left(\frac{N^{2} H}{g}\right) .
$$

We plot $B_{c}$ as a function of depth in Figure 5 (solid line). We assume $P \approx P_{g}$ in making this plot. The dashed line in Figure 5 shows the steady-state magnetic profile, arbitrarily scaled to $B=10^{10} \mathrm{G}$ at the base. For clarity, we show only models with electron-ion scattering in the crust.

In the degenerate ocean, Bildsten \& Cumming (1998) estimated the thermal buoyancy as

$$
N^{2} H^{2} \approx \frac{3 k_{B} T}{8 \mu_{i} m_{p}}
$$

allowing us to make an analytic estimate of $B_{c}$. We use equation (26) for $t_{\text {diff }} / t_{\text {accr }}$, which gives

$$
B_{c}=7 \times 10^{10} \mathrm{G} T_{8}^{1 / 2}\left[\frac{x^{3}}{g(x)}\right]^{1 / 2}\left(\frac{\dot{m}}{0.01 \dot{m}_{\mathrm{Edd}}}\right)^{-1 / 2},
$$

where we take $\Lambda_{e i}=1, g_{14}=1.9, \Gamma_{1}=4 / 3$ and $\mu_{e}=2$. For $x \ll 1$, we find $B_{c}$ is weakly dependent on depth, $B_{c} \propto x^{1 / 2} \propto y^{1 / 10}$. For $x \gg 1, B_{c} \propto x^{2}$, giving

$$
B_{c}=4 \times 10^{11} \mathrm{G} y_{10}^{1 / 2}\left(\frac{T_{8}}{3}\right)^{1 / 2}\left(\frac{\dot{m}}{0.01 \dot{m}_{\mathrm{Edd}}}\right)^{-1 / 2}
$$

in good agreement with our numerical results. 


\subsection{Doubly-Diffusive Instability}

On small enough wavelengths, thermal diffusion acts to reduce thermal gradients during the perturbation, allowing instability even for $B<B_{c}$. If the ratio of magnetic to thermal diffusivities, $\eta / \mathcal{K}$, is small, the fluid elements retain their destabilizing magnetic buoyancy, but the stabilizing thermal buoyancy is washed out (e.g., Acheson 1979). The stability criterion is as given by equation (36), except that the thermal buoyancy term is reduced by a factor $\eta / \mathcal{K}$. When there is no composition gradient, the critical magnetic field $B_{c}$ becomes $B_{c}^{\prime}=(\eta / \mathcal{K})^{1 / 2} B_{c}$. We plot $B_{c}^{\prime}$ as a dotted line in Figure 5 .

To estimate $\eta / \mathcal{K}$ in the ocean, we use the heat equation (eq. [3] ) to define a thermal time $t_{\text {therm }}=c_{p} y^{2} / \rho K=3 \kappa c_{p} y^{2} / 4 a c T^{3}$, so that $\eta / \mathcal{K}=t_{\text {therm }} / t_{\text {diff }}$. The heat transport in the ocean is by electron conduction with $K=\pi^{2} n_{e} k_{B}^{2} T \tau / 3 m_{\star}$, and we use the results of $\&$ for $t_{\text {diff }}$, giving

$$
\frac{\eta}{\mathcal{K}}=\frac{2 \times 10^{-4}}{T_{8}} \frac{\left(1+x^{2}\right)^{2}}{x^{3}} \Lambda_{e i}^{2}\left(\frac{Z}{30}\right)
$$

Taking $\Lambda_{e i}=1, g_{14}=1.9, \Gamma_{1}=4 / 3$ and $\mu_{e}=2$, the critical magnetic field is

$$
B_{c}^{\prime}=10^{9} \mathrm{G}\left[\frac{1+x^{2}}{g(x)^{1 / 2}}\right]\left(\frac{\dot{m}}{0.01 \dot{m}_{\mathrm{Edd}}}\right)^{-1 / 2}\left(\frac{Z}{30}\right)^{1 / 2} .
$$

When $x \gg 1$, we find

$$
B_{c}^{\prime}=5 \times 10^{9} \mathrm{G} y_{10}^{5 / 8}\left(\frac{\dot{m}}{0.01 \dot{m}_{\mathrm{Edd}}}\right)^{-1 / 2}\left(\frac{Z}{30}\right)^{1 / 2}
$$

a similar scaling with depth to $B_{c}$.

To summarize, we find that instability requires $B \gtrsim 10^{10} \mathrm{G}$ at the base of the ocean for $\dot{m}=0.01 \dot{m}_{\mathrm{Edd}}$, or $B \gtrsim 10^{11} \mathrm{G}$ for $\dot{m}=0.1 \dot{m}_{\mathrm{Edd}}$. We do not expect buoyancy instability to operate in the crust, as long as the rigidity of the crust is able to counteract the buoyancy force. The detailed outcome of the instability will depend on its non-linear development. However, it seems reasonable to take the critical magnetic field of equation (45) as an upper limit on the strength of any buried field at the boundary between the ocean and crust.

\section{Discussion and Conclusions}

We have investigated whether the magnetic field of a LMXB neutron star may be diamagnetically screened by the accreted matter. The magnetic profile in the outer layers is set by the competition between downwards advection and compression by accretion, and upwards transport by Ohmic diffusion. In steady-state, we have shown that the magnetic field strength decreases through the outer crust and ocean by $n$ orders of magnitude, where $n \approx \dot{m} / 0.02 \dot{m}_{\text {Edd }}$ 
(see $3_{5}$ ). The steady-state profiles are stable to buoyancy instabilities provided the magnetic field in the ocean and crust is $\lesssim 10^{10}-10^{11} \mathrm{G}$.

Our results are summarized in Figure 6. Arbitrarily choosing $B=10^{9} \mathrm{G}$ at the crust/ocean boundary, we plot the magnetic field at the surface of the ocean for different accretion rates. At large accretion rates, the magnetic field strength decreases dramatically through the ocean, and the stellar field is screened by the accreted layer. At low accretion rates, the magnetic field penetrates into the accreted layer, and screening is ineffective. The dashed and dotted lines in Figure 6 show, with and without thermal diffusion (see $\$ 6$ for the physics of this difference), the critical magnetic field needed at the top of the crust for magnetic buoyancy instability to occur in the ocean. This provides an upper limit to the strength of any buried field, since, for larger field strengths, we expect additional upwards transport of magnetic flux by buoyancy instabilities.

We have shown that for accretion rates $\lesssim 0.01 \dot{m}_{\text {Edd }}$, microscopic Ohmic diffusion prevents screening of the neutron star magnetic field by the accreted matter. At larger accretion rates, field burial may be possible; however, we stress that we have considered only a plane-parallel model with a simple field geometry. For example, consideration of a more complex field geometry in a spherical shell may indicate that additional modes of magnetic instability are available. In addition, the burial process itself is not well-understood although some calculations of the spreading of matter initially confined to the polar cap of a strongly magnetized neutron star with $B \sim 10^{12} \mathrm{G}$ have been carried out (Hameury et al. 1983; Brown \& Bildsten 1998; Litwin, Brown, \& Rosner 2001).

Our results may explain why SAX J1808.4-3658 is the only known LMXB to show pulsations in its persistent emission (upper limits to the pulsed fraction in other sources are $\sim 1 \%$; Leahy, Elsner \& Weisskopf 1983; Mereghetti \& Grindlay 1987; Wood et al. 1991; Vaughan et al. 1994). This source has similar X-ray spectral and variability properties to other LMXBs (van der Klis 2000, and references therein), but has an unusually low accretion rate. The time-averaged accretion rate is $\approx 10^{-11} M_{\odot} \mathrm{yr}^{-1} \approx 10^{-3} \dot{m}_{\text {Edd }}$ (Chakrabarty \& Morgan 1998; Bildsten \& Chakrabarty 2001), and at the peak of outburst reaches $\lesssim 0.03 \dot{m}_{\text {Edd }}$. This accretion rate is similar to that at which we expect screening to become ineffective. This leads us to suggest that perhaps most LMXBs have their dipole magnetic fields screened by the accreted matter, whereas SAX J1808.4-3658 accretes slowly enough that the magnetic field is able to rapidly penetrate the freshly-accreted material. The other faint Galactic center transients discovered with BeppoSAX (e.g., King 2000) may therefore be promising sources to search for persistent pulsations.

We have concentrated on the steady-state magnetic field profiles. However, there may be important time-dependent evolution of the magnetic field. In particular, if the magnetic field is screened, then we expect it to emerge once accretion ceases. The timescale for this depends on the depth of the screening currents (Young \& Chanmugam 1995). For the inner crust, the Ohmic diffusion time is $10^{4}-10^{5}$ years, always less than the accretion time (see Figure 9 of Brown \& Bildsten 1998). Thus we expect the relevant timescale to be the Ohmic time across the outer 
crust, which is extremely short, only 100-1000 years. It therefore seems unlikely that screening alone can explain the low magnetic fields of the millisecond radio pulsars. Potentially observable time-dependent effects may occur in those transiently-accreting systems whose accretion rate during outburst is large enough for screening to be important. After an outburst, we would expect the magnetic field profile to relax by Ohmic diffusion on a timescale comparable to the outburst duration.

Our results show that evolution of magnetic field in the outer crust is sensitive to the level of impurity there, as suggested by Schatz et al. (1999). For a primordial crust with a low level of impurity, as assumed by previous workers, phonon scattering dominates the conductivity. However, as shown by recent calculations of nucleosynthesis during $\mathrm{H} / \mathrm{He}$ burning in the atmosphere (Schatz et al. 1998, 1999; Koike et al. 1999), the crust is likely very impure. In this case, the conductivity is set by scattering from ions, leading to shorter Ohmic dissipation times than previously assumed.

We have not discussed the layer of $\mathrm{H} / \mathrm{He}$ that lies above the ocean. Because of the different composition, the Ohmic diffusion time in this layer is longer than that in the ocean. However, we find that the magnetic profile in this layer is determined by a different process, thermomagnetic drift (Urpin \& Yakovlev 1980) driven by the heat flux from nuclear burning. The evolution of the magnetic field in this layer is important for understanding Type I X-ray bursts. In particular, the drifting oscillations observed during bursts with RXTE (see Strohmayer 1999 and van der Klis 2000 for reviews) have been interpreted as due to a differentially rotating atmosphere. However, Cumming \& Bildsten (2000) pointed out that a poloidal field $\gtrsim 10^{5} \mathrm{G}$ is large enough to prevent differential rotation. Type I X-ray bursts are thus promising probes of the magnetic field in the surface layers. We address magnetic field evolution in the $\mathrm{H} / \mathrm{He}$ layers in a separate paper.

We would like to thank Phil Arras, Steve Cowley, Ramesh Narayan, Andreas Reissenegger, Chris Thompson, and Dmitrii Yakovlev for useful conversations. This research was supported in

part by the National Science Foundation under Grant PHY99-07949 and Grant AST-9800616, and by NASA via grant NAG 5-8658. L.B. is a Cottrell Scholar of the Research Corporation.

\section{REFERENCES}

Acheson, D. J. 1979, Solar Physics, 62, 23

Baiko, D. A., \& Yakovlev, D. G. 1995, Ast. Lett., 21, 702

Bhattacharya, D. 1995, in X-Ray Binaries, ed. W. H. G. Lewin, J. van Paradijs, \& E. P. J. van den Heuvel (Cambridge: Cambridge University Press), 233

Bhattacharya, D., \& Srinivasan, G. 1995, in X-Ray Binaries, ed. W. H. G. Lewin, J. van Paradijs, \& E. P. J. van den Heuvel (Cambridge: Cambridge University Press), 495

Bildsten, L. \& Chakrabarty, D. 2001, submitted to Ap. J. Letters 
Bildsten, L., \& Cumming, A. 1998, ApJ, 506, 842

Bildsten, L., \& Cutler, C. 1995, ApJ, 449, 800

Bisnovatyi-Kogan, G. S., \& Komberg, B. V. 1974, Sov. Astr., 18, 217

Brown, E. F. 2000, ApJ, 531, 988

Brown, E. F., \& Bildsten, L. 1998 ApJ, 496, 915

Chakrabarty, D., \& Morgan, E. H. 1998, Nature, 394, 346

Clayton, D. D. 1983, Principles of Stellar Evolution and Nucleosynthesis (Chicago: University of Chicago Press)

Cumming, A., \& Bildsten, L. 2000, ApJ, 544, 453

Farouki, R. T., \& Hamaguchi, S. 1993, Phys. Rev. E, 47, 4330

Geppert, U., \& Urpin, V. 1994, MNRAS, 271, 490

Goldreich, P., \& Reisenegger, A. 1992, ApJ, 395, 250

Hameury, J. M., Bonazzola, S., Heyvaerts, J., \& Lasota, J. P. 1983, A\&A, 128, 369

Hansen, C. J., \& Kawaler, S. D. 1994, Stellar Interiors: Physical Principles, Structure and Evolution (New York: Springer-Verlag)

King, A. R. 2000, MNRAS, 315, L33

Koike, O., Hashimoto, M., Arai, K., \& Wanajo, S. 1999, A\&A, 342, 464

Konar, S., \& Bhattacharya, D. 1997, MNRAS, 284, 311

Leahy, D. A., Elsner, R. F., \& Weisskopf, M. C. 1983, ApJ, 272, 256

Litwin, C., Brown, E. F., \& Rosner, R. 2001, ApJ, in press

Mereghetti, S., \& Grindlay, J. E. 1987, ApJ, 312, 727

Newcomb, W. A. 1961, Phys. Fluids, 4, 391

Potekhin, A. Y., Baiko, D. A., Haensel, P., \& Yakovlev, D. G. 1999, A\&A, 346, 345

Psaltis, D., \& Chakrabarty, D. 1999, ApJ, 521, 332

Romani, R. W. 1990, Nature, 347, 741

Romani, R. W. 1995, in Millisecond Pulsars, A Decade of Surprise, ASP Conference Series, Volume 72, ed. A.S. Fruchter, M. Tavani, \& D.C. Backer (Astronomical Society of the Pacific: San Francisco), 288

Schatz, H., et al. 1998, Phys Rep, 294, 167

Schatz, H., Bildsten, L., Cumming, A., \& Wiescher, M. 1999, ApJ, 524, 1014

Strohmayer, T. E. 1999, in X-ray Astronomy '99, Stellar Endpoints, AGN and the Diffuse X-ray Background (astro-ph/9911338)

Taam, R. E., \& van den Heuvel, E. P. J. 1986, ApJ, 305, 235 
Urpin, V. A., \& Yakovlev, D. G. 1980, Soviet Astron., 24, 425

van der Klis, M. 2000, ARA\&A, 38, 717

Vaughan, B. A., et al. 1994, ApJ, 435, 362

White, N. E., Nagase, F., \& Parmar, A. N. 1995, in X-Ray Binaries, ed. W. H. G. Lewin, J. van

Paradijs, \& E. P. J. van den Heuvel (Cambridge: Cambridge University Press), 1

Wijers, R. A. M. J. 1997, MNRAS, 287, 607

Wijnands, R., \& van der Klis, M. 1998, Nature, 394, 344

Wood, K. S., et al. 1991, ApJ, 379, 295

Yakovlev, D. G. \& Urpin, V. A. 1980, Soviet Astron., 24, 303

Young, E. J., \& Chanmugam, G. 1995, ApJ, 442, L53

Ziman, J. M. 1964, Principles of the Theory of Solids (Cambridge: Cambridge Univ. Press)

This preprint was prepared with the AAS $\mathrm{LAT}_{\mathrm{E}} \mathrm{X}$ macros v4.0. 


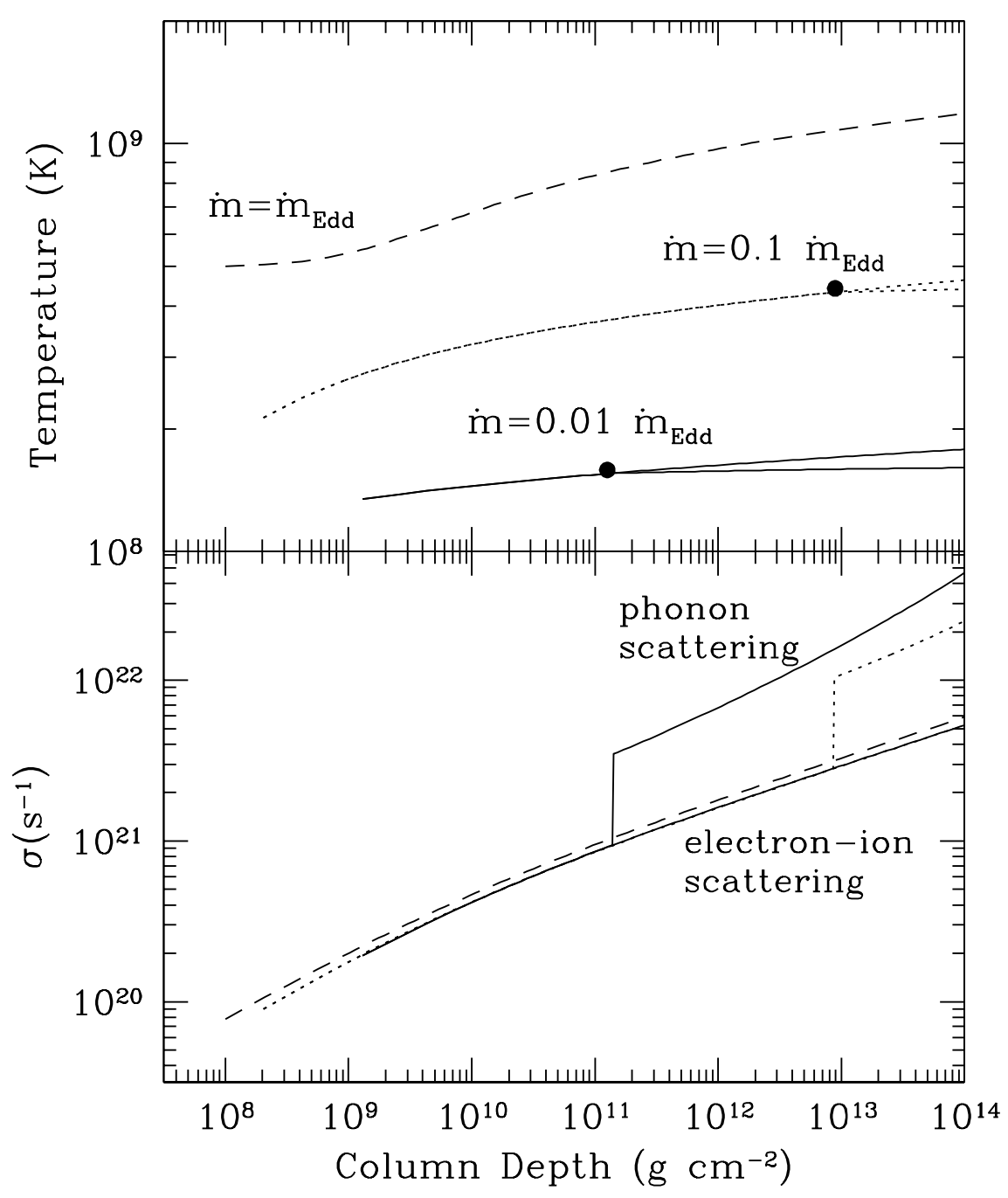

Fig. 1.- Temperature and conductivity profiles in the ocean and outer crust. We take the temperature and column depth at the top of the ocean from the X-ray burst ignition models of Cumming \& Bildsten (2000) for $\dot{m}=0.01$ (solid curves) and $0.1 \dot{m}_{\text {Edd }}$ (dotted curves), and from the steadily burning model of Schatz et al. (1999) for $\dot{m}=\dot{m}_{\text {Edd }}$ (dashed curves). We assume a single species of nuclei with $A=60$ and $Z=30$, and assume a constant heat flux of $F=150 \mathrm{keV}$ per nucleon. We indicate the ocean-crust boundary with a filled circle (the $\dot{m}_{\text {Edd }}$ model remains liquid for $y<10^{14} \mathrm{~g} \mathrm{~cm}^{-2}$ ). For 0.01 and $0.1 \dot{m}_{\mathrm{Edd}}$, the two different curves beyond the filled circle are for either electron-ion scattering or phonon scattering in the crust. 


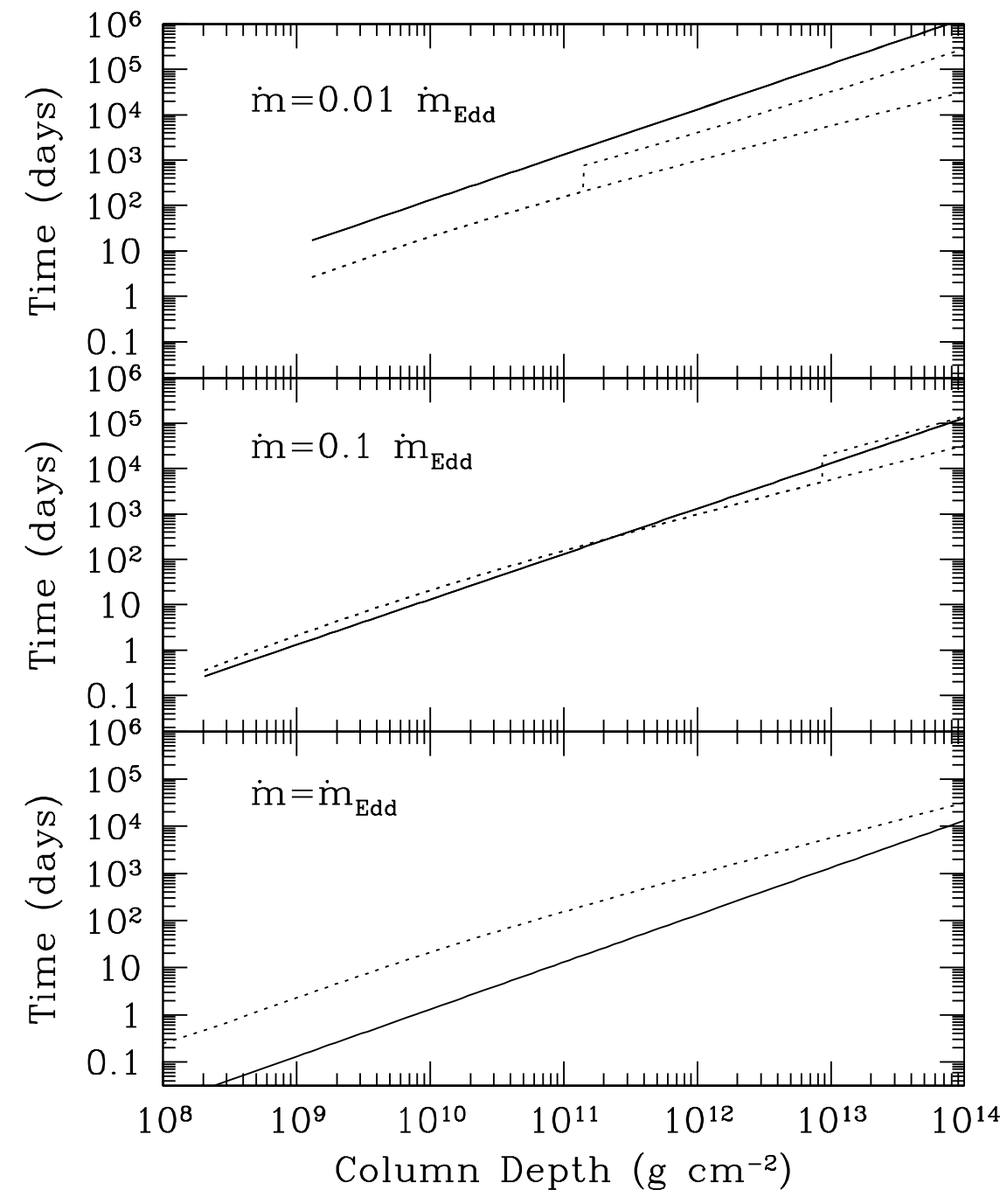

Fig. 2.- Accretion time (solid line) and Ohmic diffusion time (dotted line) across a scale height, for (top to bottom panels) $\dot{m}=0.01,0.1$ and $1 \dot{m}_{\mathrm{Edd}}$. The two solutions in the crust are for phonon or electron-ion scattering (see Figure 1). 


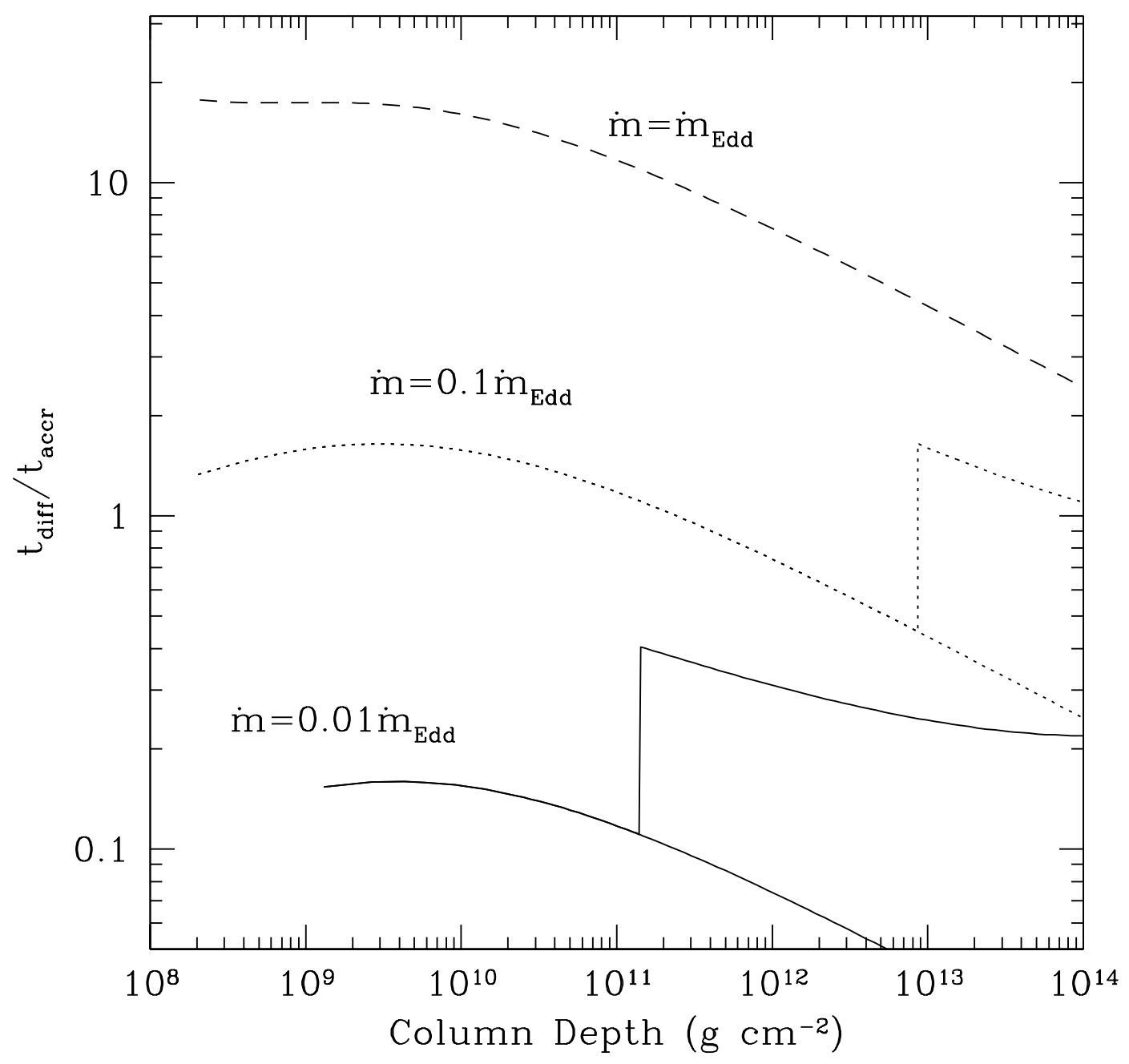

Fig. 3.- The ratio of Ohmic diffusion time across a pressure scale height $t_{\text {diff }}$ to the accretion flow time across a pressure scale height $t_{\text {accr }}$, for $\dot{m}=0.01$ (solid), 0.1 (dotted) and $1 \dot{m}_{\text {Edd }}$ (dashed line). The two solutions in the crust are for phonon or electron-ion scattering. 


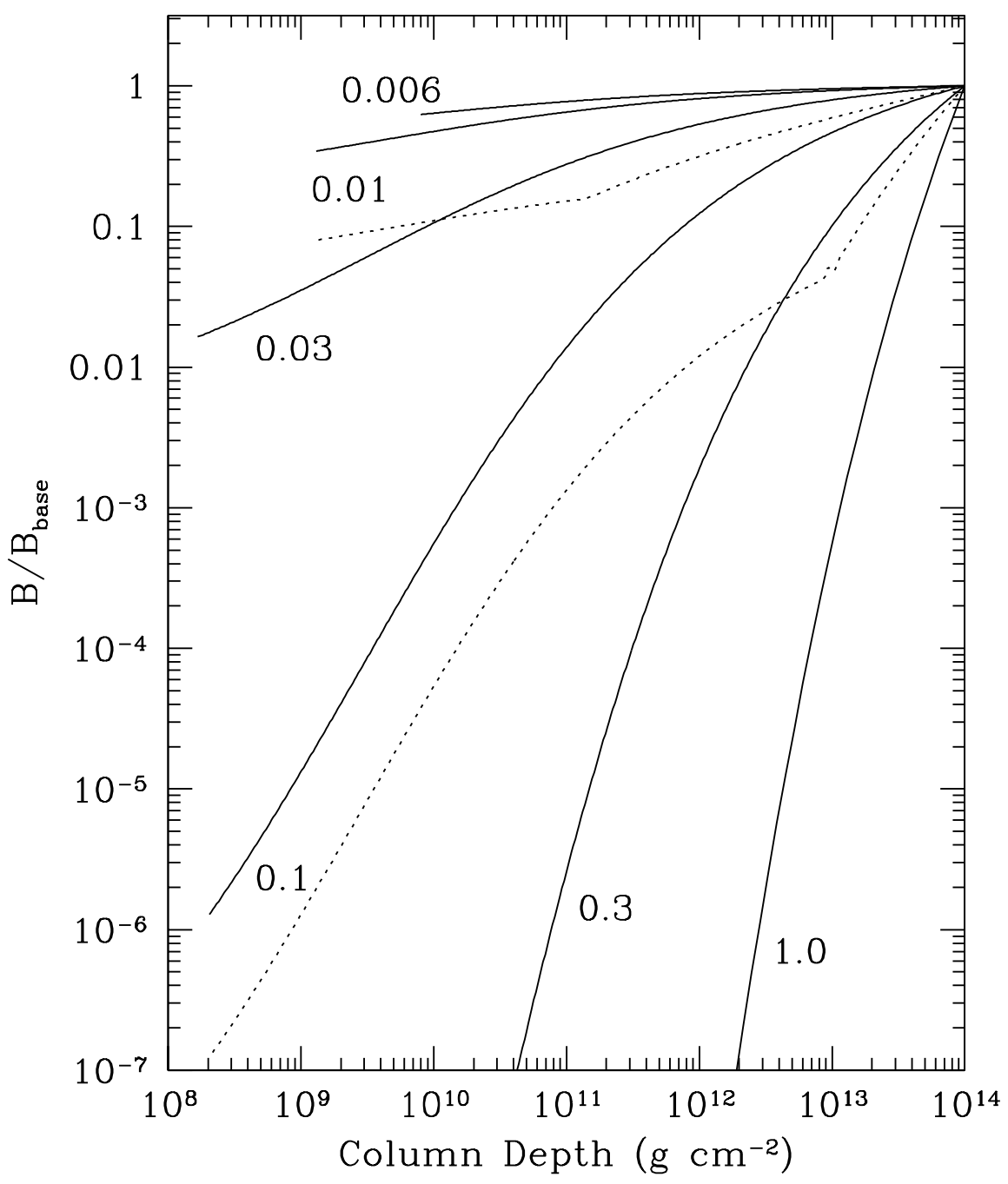

Fig. 4. - Steady-state profiles of the magnetic field, for (left to right) $\dot{m}=0.006,0.01,0.03,0.1,0.3$ and $1 \dot{m}_{\text {Edd }}$. We plot the magnetic field relative to the value at $y=10^{14} \mathrm{~g} \mathrm{~cm}^{-2}$. The solid curves show the profiles for electron-ion scattering in the crust. For $\dot{m}=0.01$ and $0.1 \dot{m}_{\text {Edd }}$, the dotted curves show the profiles for phonon scattering. 


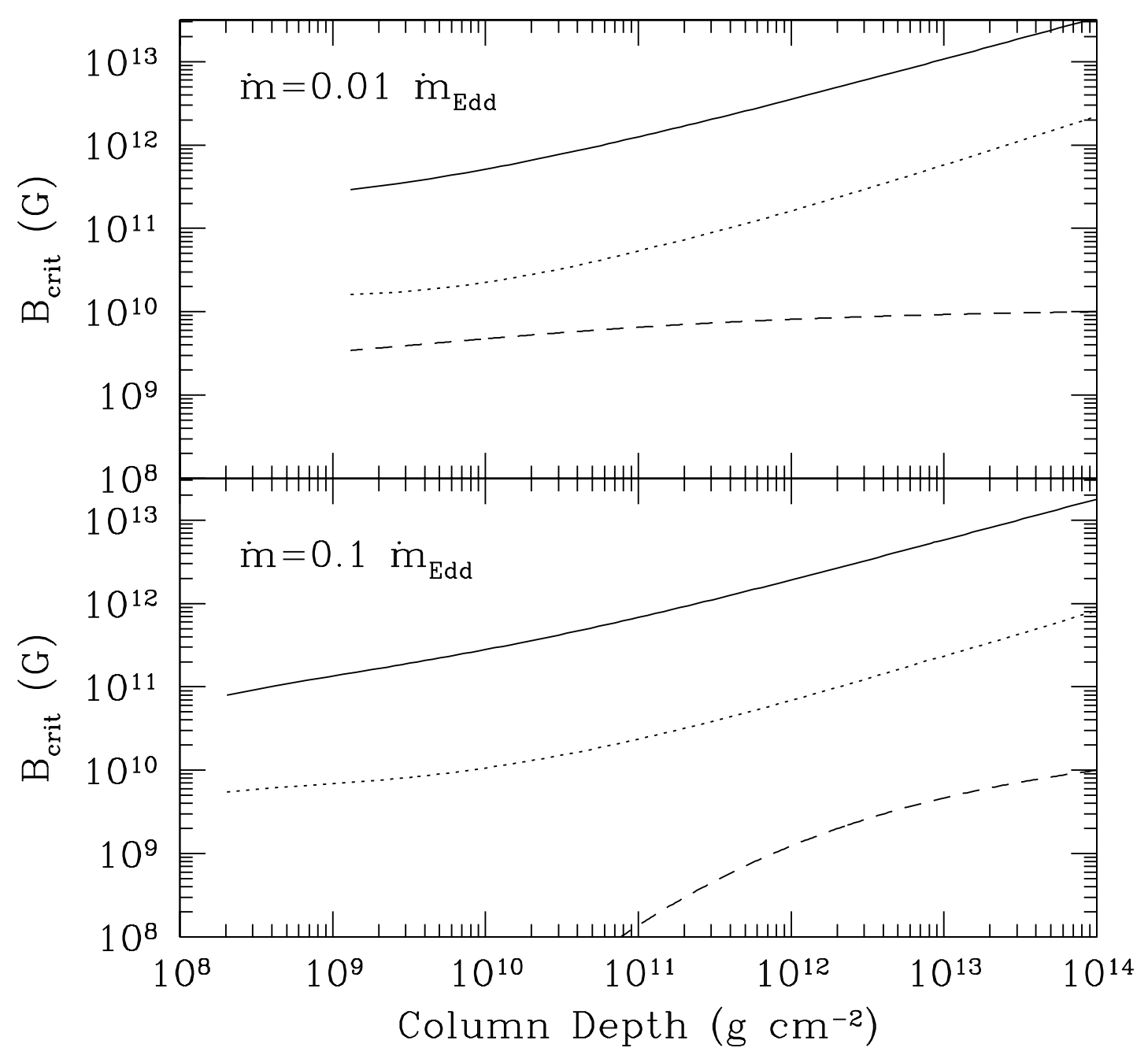

Fig. 5.- The critical magnetic field for buoyancy instability. Taking the gradient of the steadystate magnetic profile (shown as a dashed line, arbitrarily scaled to $10^{10} \mathrm{G}$ at the base), we calculate the critical magnetic field at which buoyancy instability will occur. The solid line shows the critical field ignoring thermal diffusion, the dotted line includes thermal diffusion, which reduces the effective thermal buoyancy. For clarity, we adopt only models with electron-ion scattering in the crust for this figure. 


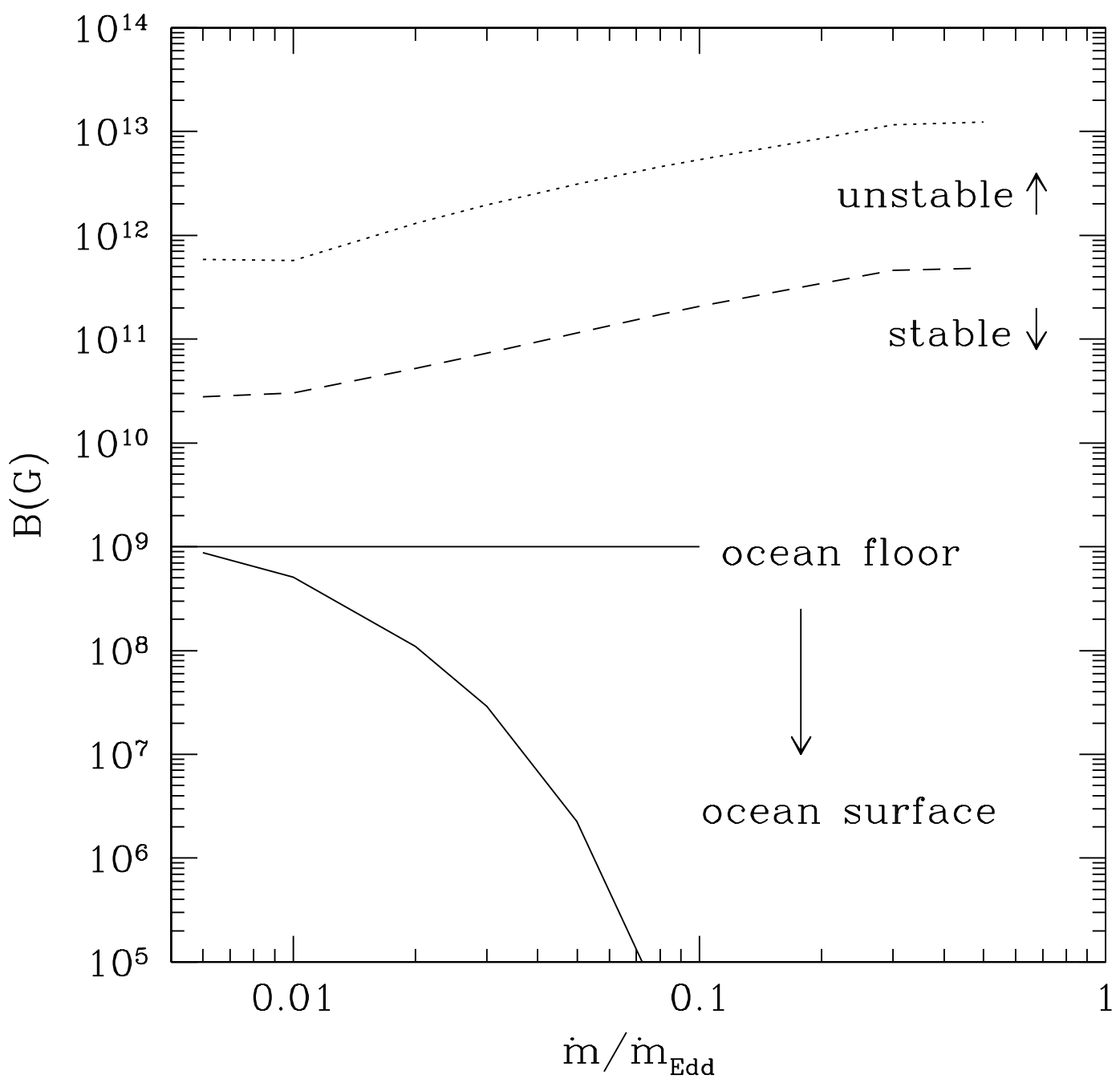

Fig. 6.- A summary of our results. The solid lines indicate the screening of the magnetic field by the ocean. Assuming a value of $10^{9} \mathrm{G}$ at the base of the ocean (upper solid line), we plot the $\mathrm{B}$ field at the ocean surface (lower solid line). The magnetic field strength decreases by roughly $\dot{m} / 0.02 \dot{m}_{\text {Edd }}$ orders of magnitude through the ocean. The dashed and dotted lines show the B field needed at the ocean floor for magnetic buoyancy instability to occur in the ocean, with and without the effects of thermal diffusion, respectively. The dashed line gives an upper limit to the strength of any buried field. 